\title{
Dual polarized open-ended waveguide using 7-layer PTFE board
}

\author{
Takashi Maruyamaa), Satoshi Yamaguchi, Tomohiro Takahashi, \\ Masataka Otsuka, and Hiroaki Miyashita
}

Information Technology R\&D Center, Mitsubishi Electric Corporation, 5-1-1 Ofuna, Kamakura, Kanagawa 247-8501, Japan

a)t-maru@ieee.org

Abstract: We propose a wideband and dual polarized open-ended waveguide for planar array antennas. The proposal forms most of the antenna structures including waveguide and feeding networks in the dielectric substrates. This yields two advantages. First, it reduces the antenna dimensions because the waveguide is filled with dielectric; this yields $30 \%$ bandwidth and short element spacing without grating lobes. Second, it simplifies the machining process, especially for the case of large scale arrays consisting of several hundred elements. We design antenna elements and a four-element array, and then fabricate the four-element array using a 7-layer board of PTFE. Though the calculated and measured reflection characteristics diverge slightly, the radiation patterns well match.

Keywords: dual polarization, open-ended waveguide, planar array, multilayer board

Classification: Antennas and Propagation

\section{References}

[1] S. Vaccaro, D. Llorens del Rio, J. Padilla, and R. Baggen, "Low cost Ku-band electronic steerable array antenna for mobile satellite communications," Proc. 5th EUCAP, pp. 2362-2366, Apr. 2011.

[2] J. M. Inclán-Alonso, A. García-Aguilar, L. Vigil-Herrero, J. M. Fernandez Gonzalez, J. SanMartín-Jara, and M. Sierra-Perez, "Portable low profile antenna at X band," Proc. 5th EUCAP, pp. 2043-2047, Apr. 2011.

[3] H. Arai and T. Izumi, "Wideband dual-polarized small tapered slot antenna for MIMO emulator,” IEEE AP-S, pp. 438-439, 2013. DOI:10.1109/APS.2013. 6710880

[4] T. Maruyama, S. Yamaguchi, T. Takahashi, and H. Miyashita, "Design and Fabrication of Wideband and Dual Polarized Open-Ended Waveguide," IEEE Trans. Antennas Propag., vol. 62, no. 9, pp. 4872-4876, Sept. 2014. DOI:10. 1109/TAP.2014.2333093 


\section{Introduction}

Active phased array antennas (APAA) are attractive for vehicular wireless communications because of their low profile and lack of moving parts [1]. However, gain loss for beam scanning is unavoidable and the many RF components raise costs excessively. Our solution is a mechanically driven planar array where all elements are excited in phase. Our goal is to realize a mechanically driven planar array yielding dual polarization and 30\% frequency bandwidth. Because the array antenna with several hundred elements is required for high gain, the antenna structure must be easy to make. Because all elements are connected to a passive beam forming network, low loss antenna elements are also required. This paper proposes solutions to meet all of these requirements.

The first candidate for an array element with wideband and dual polarization is the stacked patch antenna [2]. It has simple structure and it may achieve 30\% bandwidth. Unfortunately, relatively large conductor loss and dielectric loss are unavoidable. The second candidate is the tapered slot antenna [3]. It is suitable for array antennas because it can be fabricated on the dielectric substrate. Unfortunately, its dimension on the boresight axis is relatively long. Moreover, large scale arrays are hard to manufacture because the many substrates must be aligned in grid pattern.

We focused on the open-ended waveguide in earlier work [4]. Our design places strip lines in the substrate to feed the multiple elements. We designed the antenna and confirmed that the calculated and measured results agree well. However, it has two issues. The first one is antenna size. Waveguide size must be $0.5 \lambda_{1}$ or wider to pass the dominant mode, where $\lambda_{1}$ is the wavelength at the lower limit frequency. On the other hand, antenna spacing must be $1.0 \lambda_{\mathrm{h}}$ or shorter to avoid grating lobes, where $\lambda_{\mathrm{h}}$ is the wavelength at the upper limit frequency. Our design has difficulty in satisfying both constraints due to waveguide size. The second issue is manufacturing complexity. The design stacks multiple metal parts and dielectric substrates which raises machining and assembly costs. Additionally, it was difficult to connect the signal line from the antenna bottom to the strip line because the line must pass through metal parts and dielectric substrates. In this paper, we drastically change the antenna structure. Most antenna structures, including the waveguide and feeding circuits, are formed in the multi-layer board. We fabricate 7-layer board of PTFE substrates to reduce dielectric loss. Though PTFE multi-layer boards are not common due to their viscosity, this structure has significant advantages. The antenna dimensions are reduced because the waveguide is filled with dielectric. This achieves the $30 \%$ frequency bandwidth and the antenna spacing of $1.0 \lambda_{\mathrm{h}}$ or shorter. Additionally, when several hundred elements are required, the machining process can be reduced because etching is used. The vertical signal line from the antenna bottom to the strip line is also easily realized by through holes formed when fabricating the multi-layer board. We designed the antenna element and four-element array with antenna spacing of $1.0 \lambda_{\mathrm{h}}$ or shorter. We fabricated the four-element array. Though there was some discrepancy between the calculated and measured reflection characteristics, the radiation patterns agreed well. 


\section{Antenna structure}

The proposed element antenna structure is shown in Fig. 1(a). The antenna consists of a multi-layer board together with a metal aperture. 'L1' to 'L8' are copper layers. The 8-copper layers and 7-dielectric substrates are united to form one multi-layer board. L2 is the strip line and excitation probe layer for V polarization. L1 and L3 are its ground layers. L4 is the strip line and excitation probe layer for $\mathrm{H}$ polarization. L3 and L5 are its ground layers. Each polarization has opposite two excitation probes in a symmetrical structure. They yield small coupling between the orthogonal polarizations and low sidelobes. The strip lines of both polarizations can not be co-layered when opposite two excitation probes are connected to branched strip line, explained in Section 3, so the two feeding networks are stacked. However, this structure creates a characteristic difference between the polarizations. L7 is a slits layer for characteristic equalization. The slits act as reflectors for $\mathrm{V}$ polarization because the slits are parallel to the electric field. The electric field of $\mathrm{H}$ polarization, which is perpendicular to the slits, passes through the slits and is reflected at the antenna ground L8. This equalizes the characteristic difference between the polarizations.

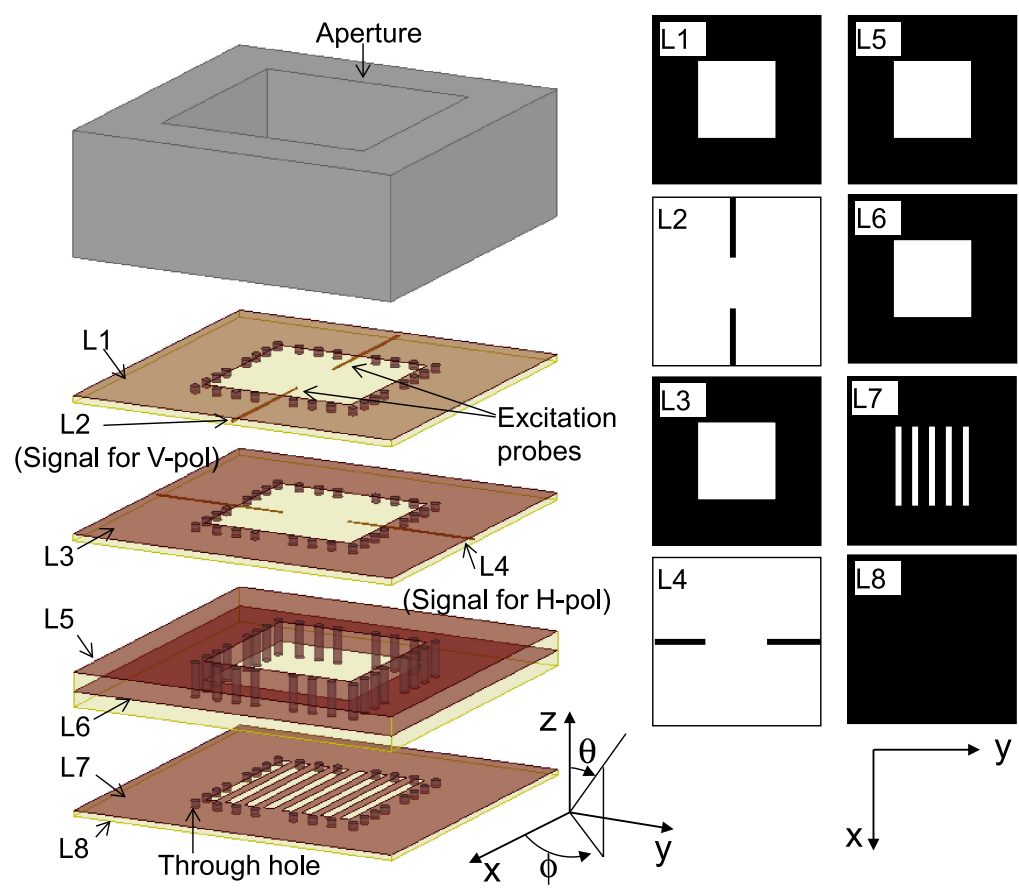

(a) Exploded perspective view

(b) Outline of copper layers

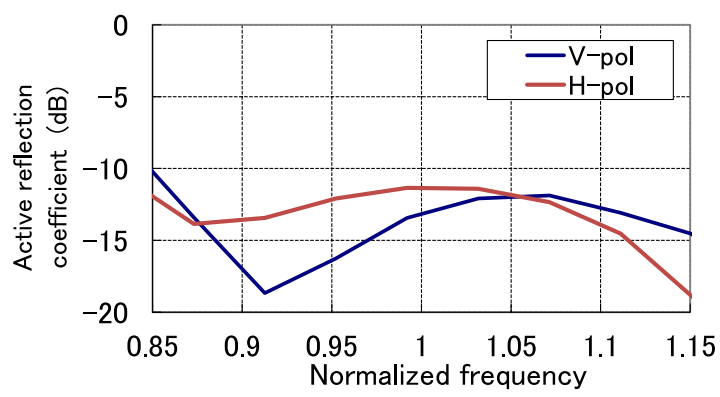

(c) Active reflection 
We designed the antenna element using HFSS, an electromagnetic field simulator. The dielectric substrate is PTFE composite with the relative permittivity of around 2.1. The calculation model has four feeding points at the edge of strip lines. The aperture size is $0.79 \lambda$, where $\lambda$ is center frequency wavelength. This corresponds to $0.91 \lambda_{\mathrm{h}}$. The waveguide size, the white square in L1, 3, 5, 6, is $0.58 \lambda$. It corresponds to $0.49 \lambda_{1}$. In the proposed antenna, the waveguide size is miniaturized because the waveguide is filled with PTFE; the remaining space holds the strip line. Its design is shown in Section 3. Through holes are placed to form the conducting walls of the waveguide. The probe length is adjusted so as to decrease reflection. The length was $0.29 \lambda$ for $\mathrm{V}$ polarization and $0.31 \lambda$ for $\mathrm{H}$ polarization.

Fig. 1(c) shows the active reflection plots. The plot of V-pol (H-pol) means the active reflection yielded when the two signal lines of L2 (L4) are excited in reversed phase. The two plots are similar due to L7 slits. The active reflection value is $-10 \mathrm{~dB}$ or lower for both polarizations in the $30 \%$ bandwidth.

\section{Design of four-element array}

We designed and fabricated a four-element array; the four elements were strip line fed. A picture of the prototype is shown in Fig. 2(a). Its side view is in (b). Though the prototype has six apertures, each polarization uses four apertures. Due to the flange size of the SMA connector at the antenna bottom, the two connectors could not be closely placed, so the four apertures used are shifted by one column for the two polarizations. This is a limitation of just this prototype. When the coaxial line is directly connected to the feeding network for a large scale array, no SMA connector is used and this shift is not required. Fig. 2(c) shows L2 feeding circuit pattern. The antenna spacing is $0.97 \lambda_{\mathrm{h}}$ even if the strip line is placed between waveguides. Because the antenna spacing does not exceed $1.0 \lambda_{\mathrm{h}}$, no grating lobe is radiated. The strip line length is adjusted so that the opposite probes are excited in reversed phase. The cable core of the coaxial line passes through the dielectric substrate and is connected to the strip line. The outer conductor of the coaxial line in the substrate section is formed by copper layers and through holes. When the SMA connector is not required for a large scale array, the cable core of the coaxial line is formed by using a through hole. This simplifies connection between the strip line and the antenna bottom. This is an additional advantage of the multi-layer board. L4 feeding circuit pattern is 90 degree rotated version of Fig. 2(c).

Reflection coefficient plots are shown in Fig. 3(a) and (b). Measured and calculated pattern shapes agree. However the measured frequency characteristics are shifted by about $5 \%$ and the measured level is higher. This discrepancy is probably due to the difference in the relative permittivity of PTFE between the calculation and the actual value. This antenna is sensitive to the relative permittivity because the waveguide between L1 to L8 in Fig. 1(a) is filled with PTFE. The actual permittivity can be estimated by comparing measured and calculated values. The discrepancy will be reduced if the design is modified by using the actual permittivity. Sum of dielectric loss and conductor loss does not exceed $0.4 \mathrm{~dB}$ within the frequency bandwidth in the calculation. Most losses occur in the strip line. This small loss may be acceptable because the subsequent beam forming 


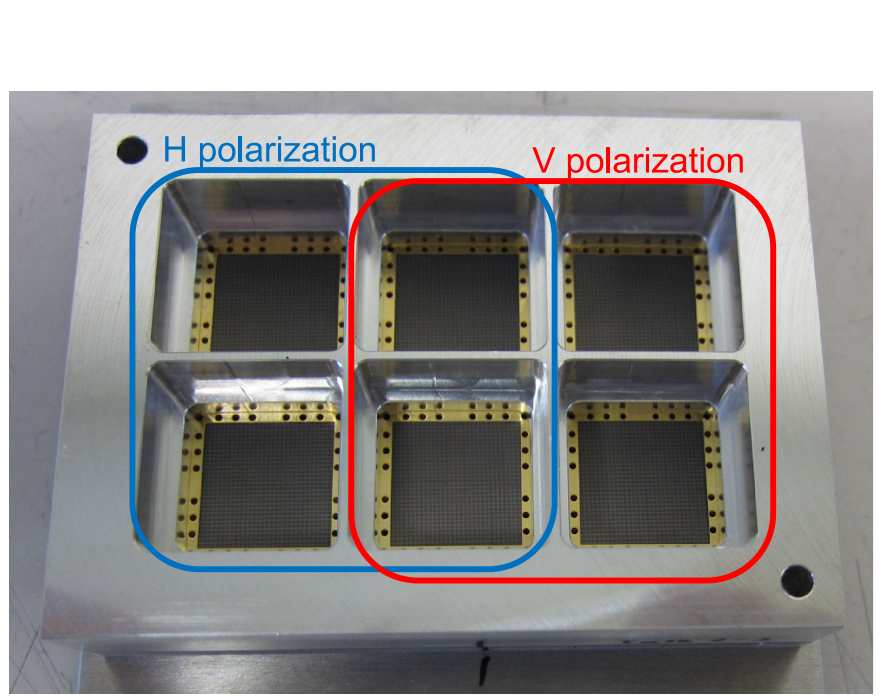

(a) Picture of four-element array (Each polarization uses four apertures)

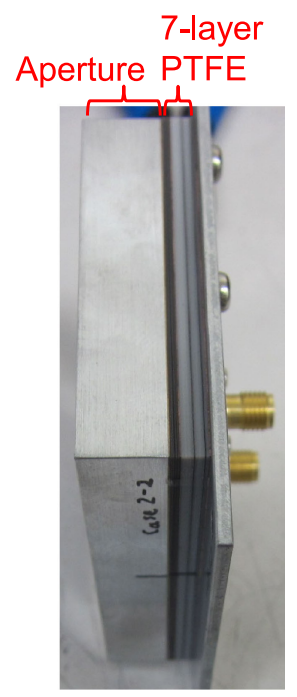

(b) Side view

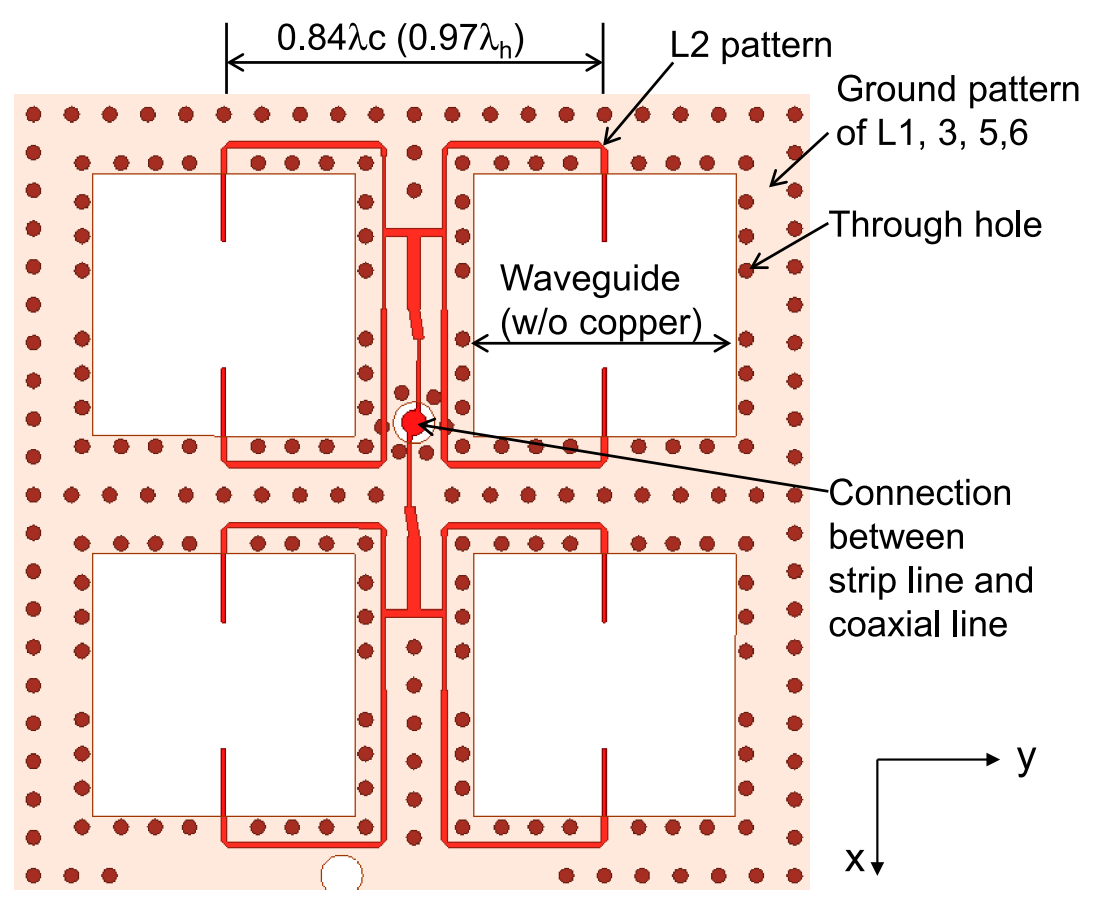

(c) Feeding circuit

Fig. 2. Four-element array

network is simplified even for large scale arrays. Fig. 3(c) and (d) are radiation patterns at the center frequency when the $\mathrm{V}$ polarization feeding circuit is excited. Fig. 3(e) and (f) are radiation patterns at the upper limit frequency; they show that no grating lobe is observed. Calculated and measured patterns approximately agreed including the cross polarization pattern. The worst cross polarization value on the boresight was $-34 \mathrm{~dB}$. Radiation patterns of $\mathrm{H}$ polarization were similar to (but rotated) those of $\mathrm{V}$ polarization. 


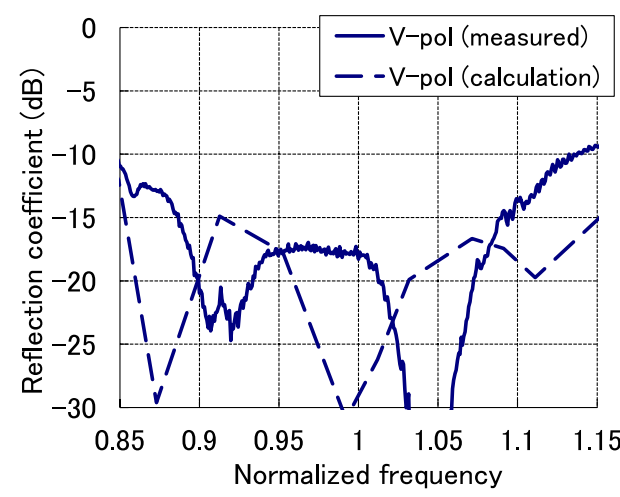

(a) Reflection of $\mathrm{V}$ polarization

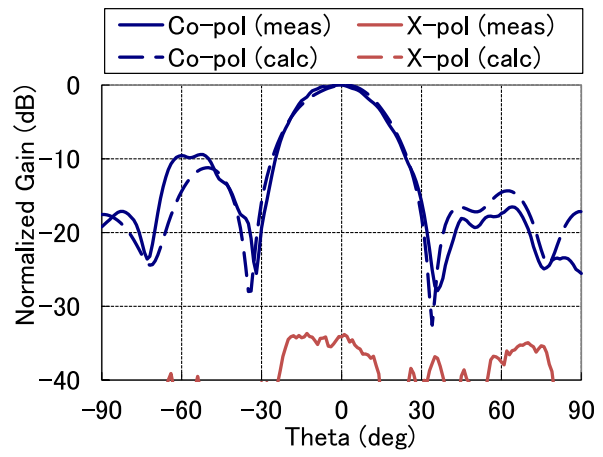

(c) Radiation pattern $(\phi=0 \mathrm{deg}, \mathrm{Fc})$

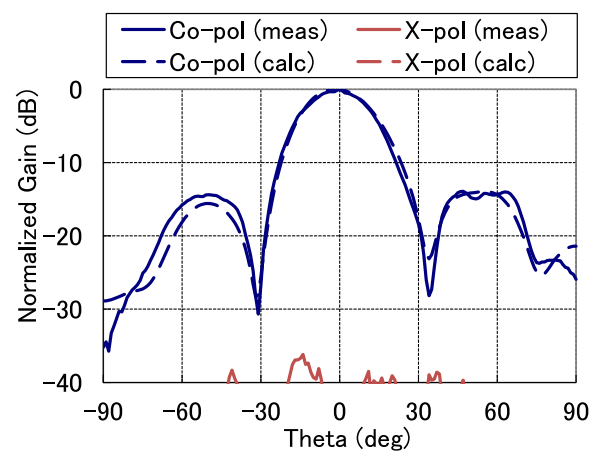

(e) Radiation pattern $(\phi=0 \mathrm{deg}, \mathrm{Fh})$

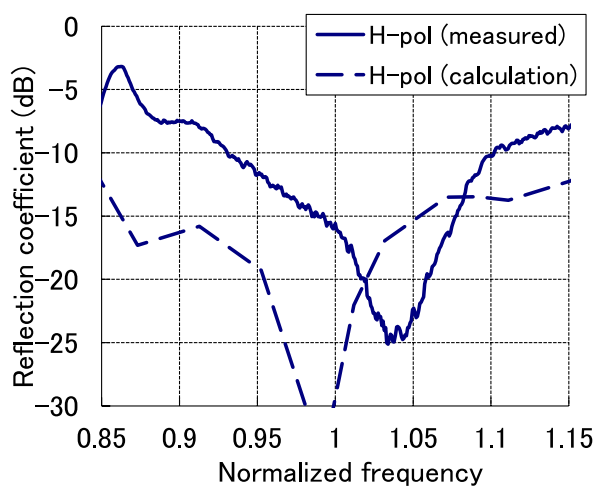

(b) Reflection of $\mathrm{H}$ polarization

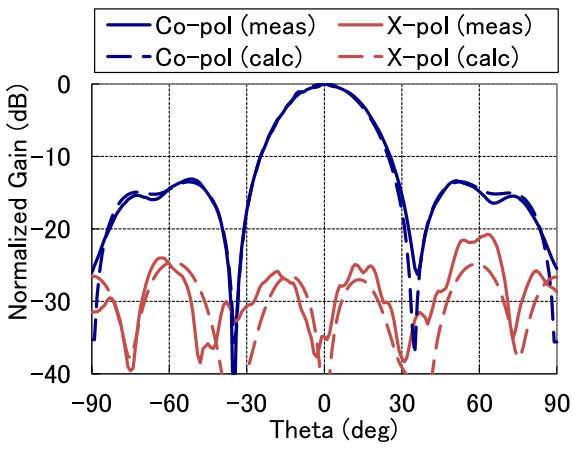

(d) Radiation pattern $(\phi=90 \mathrm{deg}, \mathrm{Fc})$

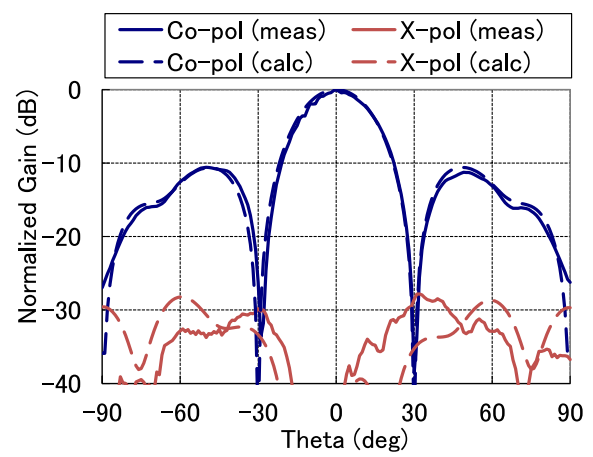

(f) Radiation pattern ( $\phi=90 \mathrm{deg}, \mathrm{Fh})$

Fig. 3. Measured and calculated results

\section{Conclusion}

We proposed a wideband and dual polarized open-ended waveguide for array antennas. Most antenna structures including strip lines and waveguide cavity are formed in a multi-layer board. A slits structure to equalize the frequency characteristics of polarizations is also included. This structure enables both $30 \%$ bandwidth and small antenna spacing because the waveguide is filled with dielectric. We designed and fabricated a four-element array with no grating lobes within the $30 \%$ bandwidth. Though the measured frequency characteristics of the reflection differed by about $5 \%$ due to a difference in the relative permittivity value assumed, the calculated and measured radiation patterns approximately agreed. 